\title{
PAIRING IN INHOMOGENEOUS SUPERCONDUCTORS
}

\author{
J. EROLES ${ }^{1,2}$, G. ORTIZ ${ }^{1}$, A. V. BALATSKY ${ }^{1}$ AND A. R. BISHOP ${ }^{1}$ \\ ${ }^{1}$ Theoretical Division, Los Alamos National Laboratory, Los Alamos, NM 87545, USA. \\ ${ }^{2}$ Centro Atómico Bariloche and Instituto Balseiro, S. C. de Bariloche, Argentina.
}

\begin{abstract}
Starting from a $t-J$ model, we introduce inhomogeneous terms to mimic stripes. We find that if the inhomogeneous terms break the $S U(2)$ spin symmetry the binding between holes is tremendously enhanced in the thermodynamic limit. In any other model (including homogeneous models) the binding in the thermodynamic limit is small or neglible. By including these inhomogeneous terms we can reproduce experimental neutron scattering data. We also discuss the connection of the resulting inhomogeneity-induced superconductivity to recent experimental evidence for a linear relation between magnetic incommensurability and the superconducting transition temperature, as a function of doping.
\end{abstract}

\section{Introduction}

Understanding the high-temperature superconducting cuprates remains a major goal in quantum many-body physics. Every conventional approach has failed to adequately explain their normal and superconducting phases. This fact, together with some new experimental data, may indeed point to the need for a new conceptual frame. In this work we propose a scenario based on three basic assumptions: 1- The superconducting state is inhomogeneous. 2- At the inhomogeneities (stripe segments), the spin-rotational symmetry is broken, providing a background for the charge carriers to form bound pairs. 3- These pairs Josephson-tunnel between stripes. In this scenario, there are (at least) two different energy scales: A lower one related to the phase coherence of the superconducting state (and therefore to $T_{c}$ ) and another related to the pairing of holes. In this regard, there are some similarities to granular superconductors.

Recent neutron, X-ray, Raman and phonon-measurements 1 , strongly suggest that at low temperatures and moderate doping the system is spatially inhomggeneous. The simplest realization of these inhomogeneities are termed "stripes." $\mathrm{I}$ In these stripes, charge clusters into nanoscale one-dimensional (1D) structures while the rest of the material displays strong antiferromagnetic correlations. There is no phase separation: the stripes are spatially separated. Note that this scenario is quite different from the BCS one, where the formation of a homogeneous superconducting state is described with a homogeneous superfluid density.

Remarkably, there is a subtle interplay between magnetism and superconductivity. In the undoped case, neutron scattering experiments show a peak at $\mathbf{k}=\mathbf{Q}=(\pi, \pi)$. At finite hole doping, this peak splits into four (at a distance $( \pm \delta, \pm \delta)$ from $\mathbf{Q})$, indicating the formation of antiferromagnetic domains. $\delta$ increases with hole concentration, suggesting that the stripes come closer. (It is believed that the concentration of holes in the stripe is nearly constant and equal to $1 / 2$.) In the insulating state the position of these four peaks is such that the stripes run along a diagonal. Upon increasing the hole concentration further, these peaks rotate by $\pi / 2$ near the superconducting transition 3 . This is evidence that the two 

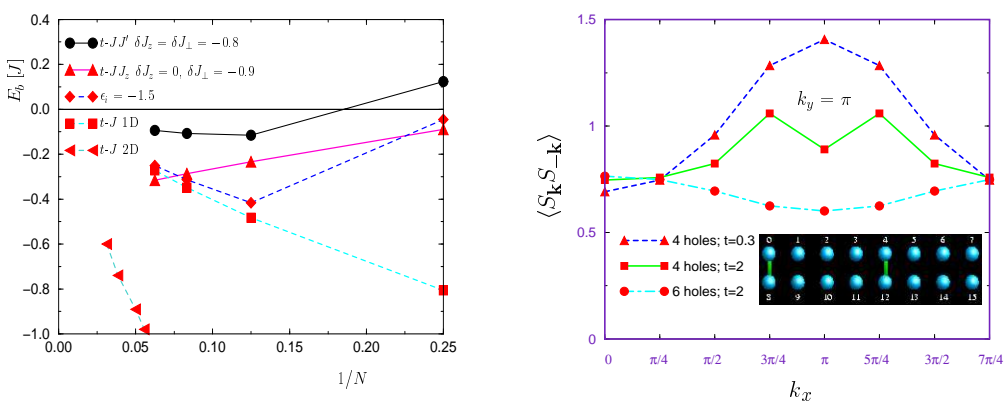

Figure 1. Left: Binding energy, $E_{b}$, for different models in $1 \mathrm{D}$ and 2D. $t-J J^{\prime}$ is a $t$ - $J$ model with two weakened bonds (with $\left.\delta J_{\perp}=\delta J_{z}<0\right)$. $t-J \epsilon_{i}$ has on site energy $\epsilon_{i}<0$ on the stripe. $t$ $J J_{z}$ has two easy-axis spin-rotational symmetry broken bonds every $P=4$ sites. In all cases $J=t=1$. Right: Spin structure factor for a $t-J J_{z}$ ladder $(8 \times 2)$ with two $\delta J_{\perp}=-0.9$ bonds in the Y-direction (see inset). The incommensurability appears only for $t$ larger than a critical value. When 6 holes are added to the system the double peak disappears and is replaced by a broad one around $\mathbf{k}=(0, \pi)$.

features (superconductivity and stripes) are inter-related. There is also experimental evidence showing that the stripes are 1D objects 3 . The relation between spin incommensuration and charge ordering has been experimentally shown in Ref.[1] where, for doping $x=\frac{1}{8}$, X-rays diffraction displays the same four peaks (with incommensuration $2 \delta$ ). On the other hand, $T_{c}$ and $\delta$ seem to be linearly related in LSCO and YBCOG: $k_{B} T_{c}=\hbar v^{*} \delta$, where $v^{*}$ defines a material-dependent velocity scale. Therefore, the only dependence of $T_{c}$ on $x$ is through $\delta(x)$. There is also experimental evidence supporting the existence of a spin-gap in these compounds 3 .

These experimental facts suggest quasi-1D objects, rich in holes, separating $\pi$ shifted antiferromagnetic domains. There has been considerable theoretical work attempting to prove that a stripe state is the low-energy state of homogeneous $t-J$ or Hubbard models 6 . There is no general consensus on this issue; it ismost probable that the stripe state is an excited state of those homogeneous models 1 . Here, we adopt a different strategy, namely introducing explicitly inhomogeneous terms in the model (which break the translational symmetry). We conclude that inhomogeneous terms breaking spinrotational invariance locally are the most efficient way to produce a bound state of two holes $\mathrm{\theta}$.

Next we will introduce our microscopic model, discuss pairing and magnetic properties of the model, and finally will make an attempt to explain the phaselocked superconducting state using a phenomenological Josephson-spaghetti model.

\section{Microscopic Model}

Our microscopic scenario 1 starts from a homogeneous $t-J$ model as background:

$$
H_{t-J}=-t \sum_{\langle i, j\rangle, \sigma} c_{i \sigma}^{\dagger} c_{j \sigma}+J \sum_{\langle i, j\rangle}\left(\mathbf{S}_{i} \cdot \mathbf{S}_{j}-\frac{1}{4} \bar{n}_{i} \bar{n}_{j}\right)
$$


where $c_{i \sigma}^{\dagger}$ creates a fermion in the space with double occupancy forbiden, $\mathbf{S}_{i}$ is the spin operator and $\bar{n}_{i}=c_{i \sigma}^{\dagger} c_{i \sigma}$ is the number operator. To mimic the stripe segments, we add inhomogeneous magnetic interactions. These inhomogeneous terms break translational invariance and spin-rotational $S U(2)$ symmetry locally:

$$
H_{\mathrm{inh}}=\sum_{\langle\alpha, \beta\rangle} \delta J_{z} S_{\alpha}^{z} S_{\beta}^{z}+\frac{\delta J_{\perp}}{2}\left(S_{\alpha}^{+} S_{\beta}^{-}+S_{\alpha}^{-} S_{\beta}^{+}\right),
$$

with $\delta J_{\perp} \neq \delta J_{z}$, representing the magnetic perturbation of a static local Ising anisotropy, locally lowering spin symmetry (named $t-J J_{z}$ model). Only a few links $\langle\alpha, \beta\rangle$ (at the stripes) have this lowered spin symmetry.

We have studied the binding energy of two holes $\left(E_{b}=\left(E_{2 \text { holes }}-E_{0 \text { hole }}\right)-\right.$ $\left.2\left(E_{1 \text { hole }}-E_{0 \text { hole }}\right)\right)$ for different $1 \mathrm{D}$ and $2 \mathrm{D}$ lattices and extrapolated to the thermodynamic limit (Fig.1 Left). We conclude that only the $t-J J_{z}$ model leads to considerable binding (we have also tried one-band Hubbard models with many different inhomogeneous terms). In the bound state, depending upon the value of $t$, the holes pair in the same or on different stripe segments (in both cases the binding energy is appreciable). It has been suggested that homogeneously breaking the spin-rotational symmetry stabilizes the stripe state 10 . Here, we argue that doing it inhomogeneously also gives an excellent hole binding mechanism. One should note that the pair is not bound to an inhomogeneity: the wave function is spread over the whole system, but more concentrated around the stripes.

This model can also explain the magnetic properties outlined above. For instance, we have calculated the magnetic structure factor in an $8 \times 2$ cluster, in which we have placed two stripes by breaking the spin-symmetry in a $Y$-link every 4 sites (the stripes are perpendicular to the $X$-axis). Although we cannot perform a good scaling here, in all cases studied the binding energy extrapolates to a significant value. The spin structure factor is shown in Fig.1 Right. The experimentally observed incommensuration appears for sufficiently high kinetic energy, $t$.

Contrary to the homogeneous $t-J$ and inhomogeneous $t-J J^{\prime}$ (where a link is weakened without breaking the spin-rotational symmetry) models, the $t-J J_{z}$ model displays a spin-gap, as seen experimentally $[$.

For a concentration near optimal doping, the stripe segments are close enough to losing their identity, suggesting the mechanism for the decrease of $T_{c}$.

\section{Phenomenological approach to inhomogeneous superfluidity}

To understand the linear relation between $T_{c}$ and $\delta(x)$, based on the microscopic model phenomenology, we introduce a Josephson Spaghetti mode 1 . This linear relation could be explained by connecting the superconducting mechanism to stripe fluctuations 2 . In the following we consider Josephson tunneling of pairs between stripe segments. The simplest mean-field model involving only the phase $\phi\left(r_{i}\right)=\phi_{i}$ of the order parameter is

$$
\mathcal{H}=\sum_{i j} J_{i j} \exp \left[i\left(\phi_{i}-\phi_{j}\right)\right], \text { where } J_{i j}=J\left(r_{i j}\right)=t_{0} / r_{i j}^{\alpha} .
$$



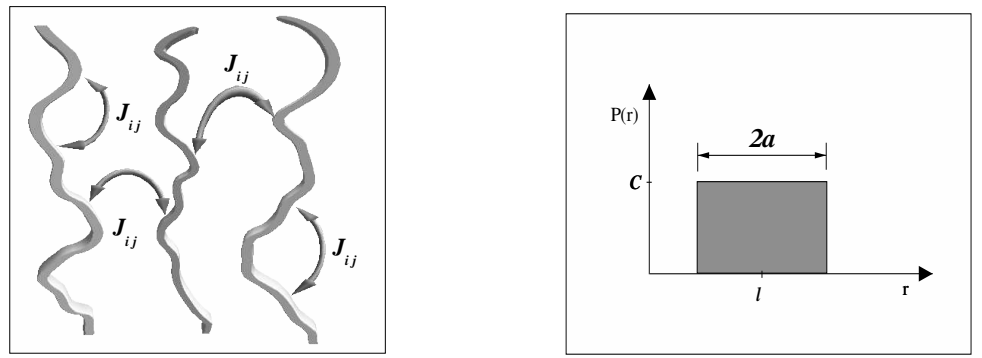

Figure 2. Schematic Josephson coupling between an assumed distribution of stripe segments (Left) and probability distribution $P(r)$ (Right). For the incommensuration $\delta$ to be observed along crystallographic $(1,0)$ and $(0,1)$ directions, the stripe-stripe distances must have average $\langle r\rangle \approx \ell=1 / \delta .\langle J\rangle$ is determined by the probability distribution $P(r)$. Clearly $P(r)$ should be centered near $\ell$, with some width $(2 a)$ from the meandering of stripes and height $C=[4 \pi \ell a]^{-1}$ (see text).

The indices $i$ and $j$ stand for coarsed grained regions where the phase is well defined (around the stripes). The Josephson coupling is an inter and intra stripe distance dependant quantity $J(r)$, and the distance $r$ is a variable with a certain distribution $P(r)$. The mean-field $T_{c}$ depends upon the Josephson coupling $\langle J(r)\rangle$. For simplicity we will take $P(r)$ as the "box" distribution depicted in Fig. 2. We find

$$
\langle J(r)\rangle=\int d^{2} r P(r) J(r)=\frac{2 \pi t_{0} C}{2-\alpha} a_{1} \ell^{2-\alpha},\langle r\rangle=\frac{2 \pi C}{3} a_{2} \ell^{3},
$$

with the constants $a_{1}$ and $a_{2} \mathcal{O}(1)$ numbers. Thus, for $\alpha=1$, we obtain the experimentally observed relation: $T_{c}(x) \simeq\langle J(r)\rangle \propto[\langle r\rangle]^{-1}=\delta(x)$.

\section{References}

1. H.A. Mook and F. Dogan, Nature 401, 145 (1999).

2. J. Zaanen and O. Gunnarsson, Phys. Rev. B 40, 7391 (1989).

3. S. Wakimoto et al., cond-mat/9908115.

4. K. Yamada et al., Phys. Rev. B 57, 6165 (1998); A.V. Balatsky and P. Bourges, Phys. Rev. Lett. 82, 5337 (1999).

5. P. Dai et al., Science 284, 1344 (1999).

6. S.R. White and D.J. Scalapino, Phys. Rev. Lett. 801272 (1998).

7. G. Seibold, C. Castellani. C. Di Castro and M. Grilli, Phys. Rev. B 58, 13506 (1998); A. Sadori and M. Grilli, cond-mat 9907447.

8. C.S. Hellberg and E. Manousakis, Phys. Rev. Lett. 83, 132 (1999).

9. J. Eroles, G. Ortiz, A.V. Balatsky and A.R. Bishop (unpublished).

10. L.P. Pryadko et al., Phys. Rev. B 60, 7541 (1999). 